\title{
Effects of movement imitation training in Parkinson's disease: a virtual reality pilot study
}

\author{
Verónica Robles-García, Yoanna Corral-Bergantiños, Nelson Espinosa, Carlos \\ García-Sancho, Gabriel Sanmartín, Julián Flores, Javier Cudeiro, Pablo Arias
}

\begin{abstract}
Background. Hypometria is a clinical motor sign in Parkinson's disease. Its origin likely emerges from basal ganglia dysfunction, leading to an impaired control of inhibitory intracortical motor circuits. Some neurorehabilitation approaches include movement imitation training; besides the effects of motor practice, there might be a benefit due to observation and imitation of un-altered movement patterns. In this sense, virtual reality facilitates the process by customizing motor-patterns to be observed and imitated.

Objective. To evaluate the effect of a motor-imitation therapy focused on hypometria in Parkinson's disease using virtual reality.

Methods. We carried out a randomized controlled pilot-study. Sixteen patients were randomly assigned in experimental and control groups. Groups underwent 4-weeks of training based on finger-tapping with the dominant hand, in which imitation was the differential factor (only the experimental group imitated). We evaluated self-paced movement features and cortico-spinal excitability (recruitment curves and silent periods in both hemispheres) before, immediately after, and two weeks after the training period.

Results. Movement amplitude increased significantly after the therapy in the experimental group for the trained and un-trained hands. Motor thresholds and silent periods evaluated with transcranial magnetic stimulation were differently modified by training in the two groups; although the changes in the input-output recruitment were similar. Conclusions. This pilot study suggests that movement imitation therapy enhances the effect of motor practice in patients with Parkinson's disease; imitation-training might be helpful for reducing hypometria in these patients. These results must be clarified in future larger trials.
\end{abstract}

\section{Keywords}

Hypokinesia; Learning; Imitative behaviour; Parkinson's disease; Virtual reality therapy; Transcranial magnetic stimulation

\author{
Abbreviations \\ (AMT) active motor threshold, (BG) basal ganglia; \\ (CV) Coefficient of variation, (EHI) Edinburgh Handedness Inventory; \\ (EG) experimental group, (FDI) first dorsal interosseus; \\ (HMD) head mounted display, $\left(\mathrm{H}_{\text {less } / \text { more-affected }}\right)$ less/more affected hemisphere; \\ (I/O curve) input-output curve, (LH) left hemisphere; \\ (MVC) maximal voluntary contraction, (M1) motor cortex; \\ (ML) motor learning, (MP) motor practice; \\ (MEP) motor evoked potential, (PD) Parkinson's disease; \\ $(\mathrm{PDp})$ patients with Parkinson's disease, $(\mathrm{CG})$ active control group; \\ (RH) right hemisphere, (RMT) rest motor threshold; \\ (SP) silent period, (TMS) transcranial magnetic stimulation; \\ (UPDRS) Unified Parkinson Disease Rating Scale, (VR) virtual reality
}




\section{Introduction}

Bradykinesia, one cardinal sign in patients with Parkinson's Disease (PDp), is formally depicted according to three dimensions: slowness, absence and reduced amplitude of movement, though currently each of these features is considered independent [1]. Dopaminergic medication is effective for bradykinesia, but hypometria is less responsive to drug treatment; for this reason hypometria should be particularly taken into account when designing a neurorehabilitation program [1].

Some of the motor impairments in PD results from dysfunction at the motor cortex (M1) intracortical networks emerging secondary to basal ganglia (BG) dysfunction [2]. The functional integrity of these networks can be evaluated by transcranial magnetic stimulation (TMS). A TMS-pulse delivered on M1 during muscle contraction generates a silent-period (SP) on the ongoing-EMG activity; this reflects the integrity of inhibitory GABAb circuits in the motor cortex [3]. Also, the motor evoked potential (MEP)amplitude induced by the stimulation increases in size with pulse-intensity, thus it is possible to build-up an I/O curve indicating the excitability of the system [4]. These explorations in PD indicate deficits in the cortical inhibitory control, reflected as a shortened SP duration and a steeper input-output (I/O) curve [2] and [4]. These deficits might be modified by motor-practice (MP) [5] and action-observation protocols [6].

Recently, a single session of action-observation tasks induced behavioral adaptations in PD [7]. Previously, movement observation had been shown to influence the subthalamic nucleus activity in conscious subjects [8]. However, this last point questions the functionality of an observation-imitation system in subjects with BG dysfunction, like PD. In this line, our group showed that PD are able to imitate (at real-time) finger-movements patterns different from their self-generated ones, resulting that imitation reduced movement variability and improved muscle recruitment [9]. These effects support the idea that several sessions of real-time movement imitation-training might induce lasting after-effects to improve motor function in PD.

This pilot-study evaluated the after-effects of an imitation therapy focused on patients' hypometria, for which we used a virtual reality (VR) system. VR facilitates the implementation of imitation protocols, allowing sensorial stimuli to be controlled and subjects' behavior to be monitored [10].

Our hypothesis is that a period of training, imitating full-amplitude finger patterns, will induce aftereffects on the patient's spontaneous movement amplitude.

\section{Material and methods}

This study was in accordance with the Declaration of Helsinki and approved by University of A Coruña Ethics Committee (CE-21/2013). Subjects signed consent forms.

\subsection{Subjects}

From a pool of 51 PDp approached in the Movement Disorders Unit of A Coruña University Hospital Complex 16 met the inclusion criteria and agreed to participate. The inclusion criteria were: idiopathic PD [11], right-hand dominance [12], and absence of: Tremulous-phenotype; dementia (MMSE score > 24 for inclusion); epilepsy antecedents; metallic implants in the head; PD-surgery; neurological diseases (other than PD); and visual-auditory-musculoskeletal dysfunctions disturbing task execution. Patients were screened with the Unified Parkinson's Disease Rating Scale [UPDRS [13]] and also with the Test of Upper Limb Apraxia [TULIA [14]]; none of the subjects displayed overt mirror-movements during this evaluation (Table 1). 
Table 1. Clinical characteristics of PD patients at baseline.

\begin{tabular}{|c|c|c|c|c|c|c|c|}
\hline Group subj & Age & Gender & EHI & Duration of PD & More affected side ${ }^{a}$ & UPDRSmotor/TOTAL & Total LEDs \\
\hline E1 & 71 & M & $\mathrm{R}-10$ & 2 & $\mathrm{~L}$ & $27 / 31$ & 300 \\
\hline E2 & 69 & M & $\mathrm{R}-12$ & 4 & $\mathrm{~L}$ & $23 / 35$ & 790 \\
\hline E3 & 62 & $\mathrm{~F}$ & $\mathrm{R}-10$ & 16 & $\mathrm{~L}$ & $28 / 31$ & 714 \\
\hline $\mathrm{E} 4$ & 66 & $\mathrm{M}$ & $\mathrm{R}-14$ & 6 & $\mathrm{~L}$ & $29 / 52$ & 1876 \\
\hline E5 & 87 & M & $\mathrm{R}-10$ & 3 & $\mathrm{~L}$ & $21 / 32$ & 1110 \\
\hline E6 & 60 & $\mathrm{~F}$ & $\mathrm{R}-12$ & 13 & $\mathrm{R}$ & $8 / 13$ & 910 \\
\hline E7 & 78 & $\mathrm{~F}$ & $\mathrm{R}-11$ & 2 & $\mathrm{R}$ & $35 / 42$ & 300 \\
\hline E8 & 57 & $\mathrm{M}$ & $\mathrm{R}-15$ & 2 & $\mathrm{R}$ & $19 / 30$ & 250 \\
\hline $\mathrm{C} 1$ & 65 & $\mathrm{M}$ & $\mathrm{R}-11$ & 4 & $\mathrm{R}$ & $19 / 33$ & 2100 \\
\hline $\mathrm{C} 2$ & 62 & $\mathrm{M}$ & R-12 & 4 & $\mathrm{~L}$ & $23 / 43$ & 1878 \\
\hline $\mathrm{C} 3$ & 76 & $\mathrm{M}$ & R-16 & 3 & $\mathrm{R}$ & $15 / 23$ & 798.5 \\
\hline $\mathrm{C} 4$ & 50 & M & R-22 & 8 & $\mathrm{R}$ & $40 / 71$ & 2676 \\
\hline C5 & 64 & M & R-13 & 15 & $\mathrm{~L}$ & $19 / 36$ & 1064.5 \\
\hline C6 & 58 & $\mathrm{M}$ & R-16 & 2 & $\mathrm{~L}$ & $21 / 31$ & 698.5 \\
\hline C7 & 75 & $\mathrm{M}$ & R-12 & 3 & $\mathrm{R}$ & $21 / 26$ & 300 \\
\hline
\end{tabular}

a 5 PDp in the EG were chiefly left-affected, 3 right-affected; in the CG 3PDp were chiefly left-affected, while 4 right-affected (Fisher's Exact Probability Test for difference in proportions $\mathrm{p}>0.05$ ).

PDp were randomly assigned in two groups: 8 subjects formed the experimental group (EG); and 8 the active-controlled group (CG). One CG subject withdrew from the study for reasons unrelated to the study; the final analyses included 15 PDp.

\subsection{Training}

The protocol evaluated the impact of 4-weeks imitation-therapy on movement hypometria in PD [15]. For this purpose, the EG and CG used a same VR system. However, while the EG imitated full-amplitude repetitive finger-tapping (FT) movements presented by the VR-avatar at three different tapping rates, the CG performed a protocol matching the same features presented to the EG (both in the spatial and temporal domain), but imitation. Thus for the CG, the VR-avatar reproduced on-line the self-paced movements executed by the subjects who were instructed to keep a full-amplitude (spatial domain) and follow three different rates (temporal domain). This way, we evaluated the effects of imitation-MP vs. MP alone.

The EG imitated the avatar's movement (which was customized by the experimenter); for the CG the VR captured patients' self-movements and the avatar displayed them in real-time. In all cases the VR avatar was presented in 1st person's perspective and observed through a head mounted display (HMD).

The amount of weeks/protocol, sessions/week, and practice and rest-periods/session, were the same for both groups: they trained 3 sessions per week, for 4 weeks. Each session lasted $\approx 25$ min during the first two weeks and $\approx 35$ min the last two weeks (Fig. 1A). The experimenter corrected on-line subject's performance (phase locking and movement amplitude), up to 4 times/session in both groups. The effect of the training was evaluated before (PRE), after (POST) and 2-weeks after the training period (POST-2). 


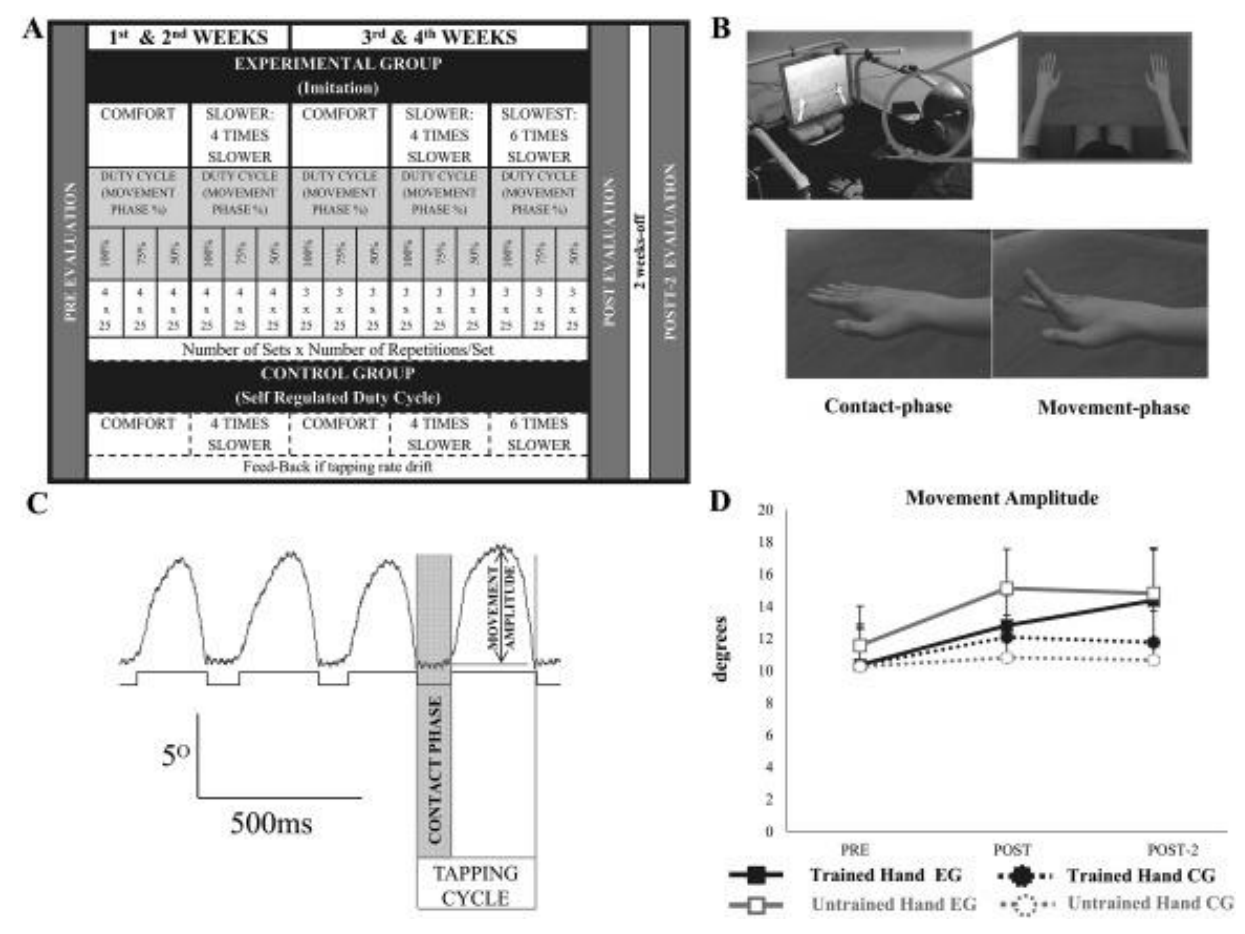

Fig. 1. Details of methods and results on hypometria. (A) Intervention protocol and evaluations times for the EG and the CG. (B) VR setting and interface. (C) Signal processing of kinematic variables. (D) The amplitude of the finger movements was significantly increased after the imitation training (EG) in both hands (see text for details).

Sessions were executed at the same time of the day; PDp were trained on-therapy (at patients' selfreport schedule for optimal drug-effect) since it is now believed that the effectiveness of neurorehabilitation protocols depends on the dopamine available at the time of training [16]. Both groups executed only with their dominant hand (right); though the effect of training was evaluated bilaterally.

\subsection{Experimental group}

The subjects were asked to "imitate avatar's FT as close as possible". The avatar's movement to be imitated displayed a functional full-amplitude index extension (and retraction) at 3 different tapping rates, presented in a randomized order (Fig. 1A-B): i) one rate matched each subject's comfort rate acquired at PRE. ii) a rate slower (4 times slower than comfort), and iii) in the last 2-weeks the slowest rate (6 times slower than comfort). The duty-cycle of the full-amplitude pattern to be imitated (relation movementcontact phase durations) were $100 \%, 75 \%$ and $50 \%$ (i.e., $75 \%=3 / 4$ of cycle moving phase; $1 / 4$ contact phase). For each session of the 1 st and 2 nd weeks the subjects imitated 6 patterns $(2$ rates $\times 3$ duty-cycles) 4 times (blocks); therefore executing 24 trials, each including 25 movement cycles. In 3rd and 4th weeks we included the slowest rate, but the number of blocks was reduced to 3, thus including 9 patterns (3 rates $\times 3$ duty-cycles), for a total of 27 trials/session ( Fig. 1A).

\subsection{Control group}

The CG practiced in the same VR as the EG. Subjects were instructed to "perform full-amplitude finger-tapping while watching to the VR hand that will move as yours, the experimenter will guide you to get the different rates". In order to do this, the experimenter, who wore earphones, listened to a metronome to check the frequencies (comfort, also acquired at PRE; slower; and slowest) and modulated the subjects' tapping-rate by verbal commands to get the corresponding rate before starting each trial. Once the rate was established, the trial started with no further consigns, except in the case tapping rate drift within the trial. In such case, the experimenter corrected subject's performance (rate and movement amplitude) up to 4 times/session in both the EG and CG. 


\subsection{Evaluations}

PDp were evaluated the day immediately before and after the training-period (PRE and POST), and after 2 weeks without MP (POST-2). Evaluations were off-therapy, after overnight withdrawal of antiparkinsonian medication $(>12 \mathrm{~h})$. PDp were evaluated off-therapy to test the effect on the "true" parkinsonian functionality and to avoid different levels of motor fluctuations within/between the three testing sessions.

Evaluations began with the study of cortico-spinal excitability, followed of motor execution (both hemispheres and hands). We used MatLab customized programs to extract the data (MatLab, The Mathworks Ltd).

\subsection{Clinical motor performance}

\subsubsection{Finger-tapping test}

During evaluations patients tapped at comfort-rate and at maximum movement amplitude (no imitation) in real environment (REAL) and VR (3 trials of 50 cycles each hand; in a randomized order). VR system validation and description is shown elsewhere [17].

We recorded the cycle duration ( $m s$ ) and movement amplitude (grades) and calculated their coefficients of variation $\left(C V_{\text {cycle-duration }}\right.$ and $C V_{\text {amplitude }}(\%)$. We used a Biometrics DataLink system (Biometrics Ltd. UK) with a metal plate and a single axis goniometer F35 (sampled at $1 \mathrm{KHz}$ and placed on the dorsal side of the index metacarpo-phalangeal joint; Fig. 1C). While evaluating one hand, recordings from the other hand were obtained to detect overt mirror movements [18].

\subsection{Cortico-spinal excitability}

TMS-MEP's were recorded from the first dorsal interosseus (FDI) muscle. TMS was applied on M1 using a $70 \mathrm{~mm}$ figure-of-eight coil, positioned tangentially to the skull and inducing currents in a posteroanterior direction. Single monophasic TMS-pulses (Magstim $200^{2}$ ) were delivered to the cortical area corresponding to FDI; the two hemispheres were explored.

MEPs were amplified $\times 1000$ and filtered at $3-3000 \mathrm{~Hz}$. Data were sampled at $10 \mathrm{kHz}$ and analyzed using a CED $1401 \mathrm{~A} / \mathrm{D}$ and Signal 4 software (Cambridge, UK).

Firstly, we determined the motor thresholds at rest (RMT) and during activation (AMT; at $\approx 10 \%$ of the maximal voluntary contraction MVC). The RMT was defined as the minimum intensity evoking a liminal response (of about $50 \mu \mathrm{V}$ ) in $50 \%$ of 10 consecutive trials with the muscle at rest [19]. Likewise, for the AMT the responses required were $\approx 200 \mu \mathrm{V}$. RMT and AMT were expressed as normalized change from Pre-values.

Subsequently, we registered the I/O $\mathrm{O}_{\mathrm{REST}}$, at intensities of 110-120-130-140\% RMT. Then, the $\mathrm{I} / \mathrm{O}_{\text {ACtIVATION }}$ at intensities of 120 and $150 \%$ AMT (just two intensities to avoid fatigue), and their silent periods (SP). TMS-pulse frequency was $0.16 \mathrm{~Hz}$, delivered repeatedly up to acquire 5 MEPs for each of the stimulation intensities with no observable background activity (for $\mathrm{I} / \mathrm{O}_{\mathrm{REST}}$ ); and with a contraction $\approx 1 / 3 \mathrm{MVC}$ for the $\mathrm{I} / \mathrm{O}_{\text {ACTIVATION }}$ (feed-back provided). At each evaluation time-point, MEP-amplitudes (peak-to-peak) were normalized by the average of the MEPs registered at the lowest intensity, considering all subjects in both groups. SP durations were determined by a blinded and experienced researcher from the TMS artifact to the reappearance of EMG-background activity, and a SP-ratio was calculated (SPratio $=$ SPduration $\left._{150 \% \mathrm{AMT}} / \mathrm{SPduration}_{120 \% \mathrm{AMT}}\right)$. Subsequently, this ratio was normalized as in the previous variable.

In all cases the EMG-background in the $70 \mathrm{~ms}$ prior to stimulation was recorded to statistically compare levels of muscle pre-activity.

\subsection{Statistical analysis}

Intra-rater reliability during the determination of the SP duration was evaluated by the intra-class correlation coefficient (ICC) and its $95 \%$ confidence interval. This was done on 100 randomly chosen SPs, which durations were determined twice. 


\subsection{Baseline evaluations}

Initially, we analyzed the EG and CG performance at PRE. For each variable we applied an ANOVA with repeated measures $\left(A N O V A_{R M}\right)$. A between-subjects factor "Group" included levels (EG and CG) to evaluate if motor execution was different at PRE in the two groups, we considered the within-subjects factors "Hand" (trained and untrained hand) and "Environment" (REAL and VR).

A student-t test compared the differences of the SP-ratio between groups at PRE for each hemisphere.

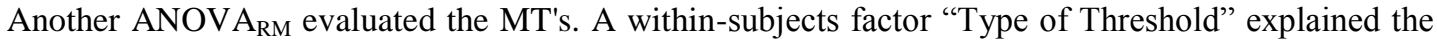
difference between AMT and RMT (2 levels); the between-subjects factor (Group) revealed if both groups differed in their thresholds.

The differences in the I/O (and EMG background) at PRE between groups were evaluated by another $\mathrm{ANOVA}_{\mathrm{RM}}$. For each hemisphere the within-subjects factor "Intensity" considered the amplitude of the $\mathrm{MEPs}_{110-140 \%} \mathrm{I} / \mathrm{O}_{\mathrm{REST}}$; the between-subjects factor was Group (EG and CG). The model was the same for $\mathrm{I}_{\mathrm{O}} \mathrm{O}_{\mathrm{Activation}}$, but the factor "Intensity" had just two levels, $\mathrm{MEP}_{120 \%}$ and $\mathrm{MEP}_{150 \%}$. Excitability variables were evaluated in a left/right hemisphere basis (LH, RH), and also for more/less affected hemispheres ( $\left.\mathrm{H}_{\text {more-affected }}, \mathrm{H}_{\text {less-affected }}\right)[20]$.

\subsection{Effects of training}

Next, we evaluated POST training effects, and recovering after a follow-up (POST-2). The ANOVA $_{R M}$ models were similar as before but adding an extra within-subjects factor "Time-point Evaluation" (levels: PRE, POST, POST-2).

Normality was tested by Kolgomorov-Smirnov for one-sample. Univariate ANOVA $A_{R M}$ was used and Greenhouse-Geisser correction applied in case of sphericity violation. Graphs show the mean and 1 SEM. Significance was set at $\mathrm{p}<0.05$.

\section{Results}

Intra-rater consistency in determination of SP duration was ICC $=0.97$ [95\%CI: 0.96-0.98]. Groups were not significantly different at PRE. For all the kinematic and cortical excitability variables Group

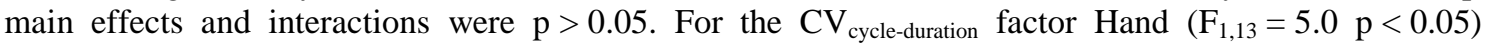
indicated a greater variability in the frequency of tapping for the non-dominant hand.

\subsection{Effects of training}

\subsubsection{Hypometria}

The analysis of the movement amplitude when considering the three evaluation time points revealed that the execution was significantly different in both groups $\left(F_{1,13}=7.7 p<0.05\right)$.

We split analyses by group and observed a main effect of "Time-point Evaluation" only in the EG, indicating that the movement amplitude was significantly increased after the imitation-training $\left(\mathrm{F}_{2,14}=5.4\right.$ $\mathrm{p}<0.05$; Fig 1D, solid-lines). The increase from PRE to POST (post-hoc $\mathrm{p}<0.05$ ) remained at POST-2 (post-hoc $\mathrm{p}<0.05$ vs. PRE). The EG's increase in amplitude was not different in both environments (REAL and VR); and very remarkably it was not different for the trained and un-trained hand $(\mathrm{p}>0.05$ for interactions; Fig 1D black and grey-lines).

The movement amplitude after the training in the CG did not change significantly ( $\mathrm{p}>0.05$ for main effects and interactions; Fig 1D, dotted-lines).

The cycle duration, $\mathrm{CV}_{\text {cycle-duration }}$ and $\mathrm{CV}_{\text {amplitude }}$ did not change with interventions ( $\mathrm{p}>0.05$ for main effects or interactions).

\subsection{Cortico-spinal excitability after intervention}

\subsubsection{Motor thresholds}

The training protocols had a different effect for the normalized thresholds of the LH in both groups; this effect was significant $F_{2,26}=7.3 p<0.05$. We split analyses for each group to understand the specific effect of the two training protocols. For the EG, the effect of training was not significant, with no 
interaction with the type of threshold (Fig. 2A.1, results pooling MT's). In the CG, MP (without imitation) decreased significantly the MT's of the LH $\left(\mathrm{F}_{2,12}=6.8 \mathrm{p}<0.05\right)$; post-hoc PRE vs. POST-2 were $\mathrm{p}<0.01$ for the AMT and RMT ( Fig. 2A.1, AMT and RMT represented pooled).

A

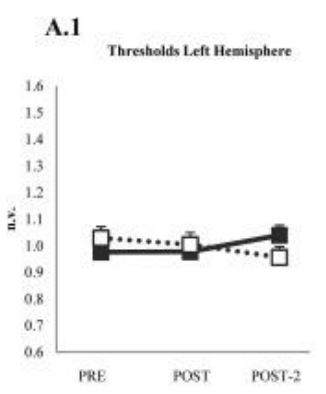

A.3

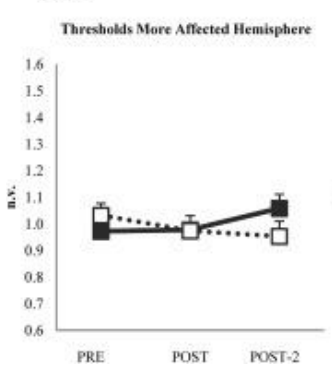

A.2

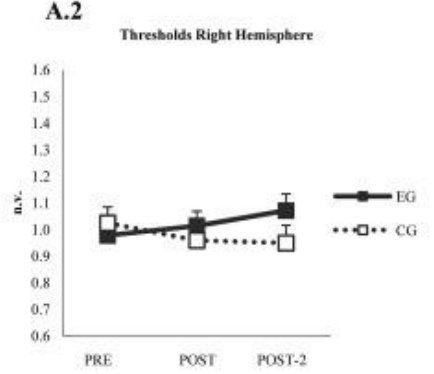

A.4

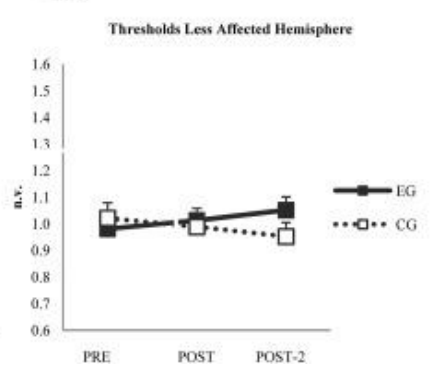

B
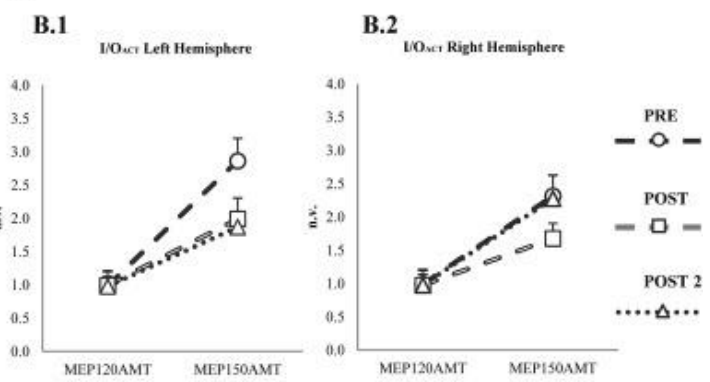

B.3

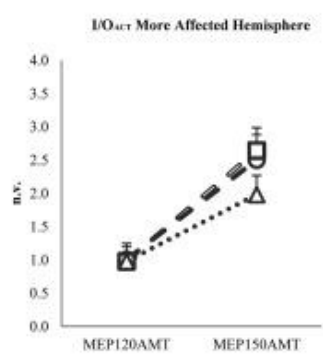

B.4

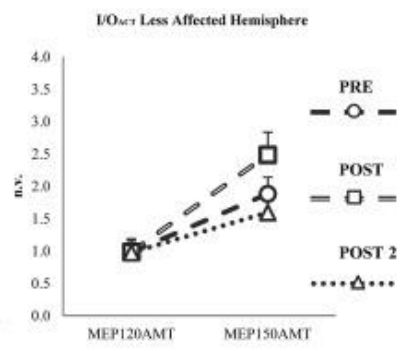

Fig. 2. Training Effects on Motor Thresholds (A) and $\mathrm{I} / \mathrm{O}_{\mathrm{Activation}}(\mathrm{B})$. Training effects on MT's where significantly different in both groups as shown in the different direction of changes after MP or MP-imitation; this was seen in analyses by hemisphere side (A.1 and A.2), or hemisphere affectation (A.3 and A.4). AMT and RMT are represented pooled, due to absence of significant interaction between Kind of Threshold and Evaluation Time Points. Values in the Y axis represent the normalized unit value (n.v.). The effect of training in $\mathrm{I} / \mathrm{O}_{\mathrm{ACT}}$ was never different in both groups (groups shown pooled) and the slope of recruitment was reduced in the LH but not in the RH (B.1 and B.2). When we considered more and less affected hemispheres (B.3 and B.4) the slope was reduced only in the less affected hemisphere at the end of the follow-up period (see text for details of the analysis in pairs).

Likewise, in the RH, the training changed MT's in a significantly different manner for both groups $\left(\mathrm{F}_{2,26}=5.1 \mathrm{p}<0.05\right.$; Fig. 2A.2). However, analyses by group never revealed a significant change comparing the values of three testing times.

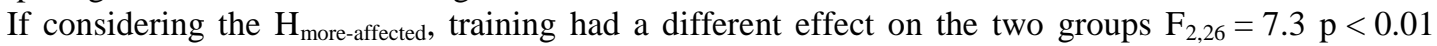
(Fig. 2A.3). In the EG, the thresholds increased significantly with training $\mathrm{F}_{2,14}=4.7 \mathrm{p}<0.05$; post-hoc indicated the effect for AMT and RMT focused at POST-2 ( $p=0.05$ vs. PRE; $p=0.06$ vs. POST). CG's thresholds were reduced significantly $\mathrm{F}_{2,12}=3.9 \mathrm{p}=0.05$, and the effect in pairs was significant for PRE vs. POST-2 (post-hoc $\mathrm{p}<0.05)$.

For the $\mathrm{H}_{\text {less-affected }}$ (Fig. 2A.4) the effect of training also differed in both groups $\mathrm{F}_{2,26}=4.2 \mathrm{p}<0.05$. EG's were unaltered; CG's reduced thresholds over time $\mathrm{F}_{2,12}=3.9 \mathrm{p}=0.05$, but none of the post-hoc comparisons in pairs were significant.

RMT was always higher than AMT $(\mathrm{p}<0.001)$, but both were affected by training not in a significantly different way (i.e., graphs are shown pooling thresholds).

\section{3. $I / O$ recruitment in activation}

For the $\mathrm{I} / \mathrm{O}_{\mathrm{ACTIVATION}}$ of the $\mathrm{LH}$ we observed the typical increase in the MEP-amplitude with increasing intensity $\left(\mathrm{F}_{1,13}=49.6 \mathrm{p}<0.001\right.$; this was similar in the rest of hemispheres analyses, ie., $\mathrm{RH}$, $\left.\mathrm{H}_{\text {more-affected, }} \mathrm{H}_{\text {less-affected }}\right)$. LH-I/O $\mathrm{O}_{\text {ACTIVATion }}$ was significantly modified by MP $\left(\mathrm{F}_{2,26}=8.7 \mathrm{p}<0.01\right)$; the effect is manifested in a less steep slope, borderline significant at POST (post-hoc; $\mathrm{p}=0.06$ ) and significant POST-2 (post-hoc; p < 0.05), compared to PRE. The effect was not differently expressed in 
the EG and $\mathrm{CG}\left(\mathrm{F}_{2,26}=0.8 \mathrm{p}>0.05\right.$, the graphs show both groups pooled) (Fig. 2B.1). Fig. 2B.2 shows the training effect in the RH (not significant).

In the $\mathrm{H}_{\text {less-affected }}$ (but not in the $\mathrm{H}_{\text {more-affected }}$ ) training reduced the $\mathrm{I} / \mathrm{O}_{\text {ACTIVATION }}$ slope $\mathrm{F}_{2,26}=4.4$ $\mathrm{p}<0.05$; the effect was detected between POST and POST-2 (post-hoc $\mathrm{p}<0.05)$ and was not different for the two groups ( $p>0.05$ ). Fig. 2B.3 shows $\mathrm{H}_{\text {more-affected }}$; $2 \mathrm{~B} .4 \mathrm{H}_{\text {less-affected. }}$

\subsection{Silent period ratio}

In the LH (Fig. 3A.1), the SP-ratio change with training was borderline different for the two groups $\left(\mathrm{F}_{2,26}=3.0 \mathrm{p}=0.06\right)$; effects on RH SP-ratio is shown in Fig. 3A.2 for comparison. Effects in LH are explained by a significant change in the EG $\left(\mathrm{F}_{2,14}=3.9 \mathrm{p}<0.05\right)$, which is absent in $\mathrm{CG}\left(\mathrm{F}_{2,12}=0.5\right.$; $\mathrm{p}>0.05$ ); follow-up analysis indicated that the SP-ratio increased significantly at POST in the EG ( $\mathrm{p}<0.05$; post-hoc), but it returned to baseline-levels after two-weeks with no training. Fig. 3A.3 and A.4 illustrate results by $\mathrm{H}_{\text {affectation }}$ (not significant).

A
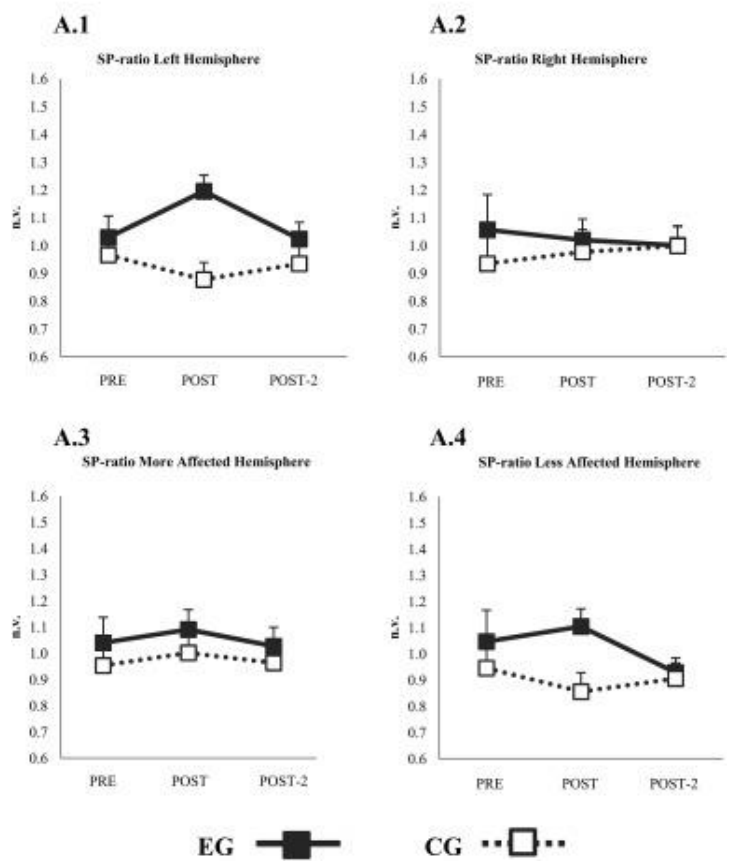

Fig. 3. Training Effects on SP-ratios. A.1. LH SP-ratio of the EG was significantly increased at POST, and returned to baseline levels at POST-2; SP-ratio of the CG was not modified, (see text for details). SP-ratios of the $\mathrm{RH}$ (A.2), $\mathrm{H}_{\text {more-affected }}$ (A.3), $\mathrm{H}_{\text {less- }}$ affected (A.4) did not change (the unit is equivalent to the corresponding SP-ratio at PRE).

For the rest of analyses main-effects and interactions were not significant. See also supplementary figures. 


\section{Discussion}

The results of this pilot-study indicate that the imitation of full-extension movements prompts visuomotor adaptations which reduce PDp's hypometria. Visual information was provided in the EG and CG (self-generated movements for the latter), but it seems that imitation of full-amplitude movements made a difference from MP alone. Previous results endorsed the idea that imitation can prompt online movement adaptations in PDp [9], now we show that imitation therapy has an offline effect in movement amplitude. EG's hypometria was reduced at virtual and real environments testing. This is of importance since ecologic validity of VR lays in the transfer of trained skills to the real world [17] and [21].

In addition, we show that 4-weeks imitation-training is enough to acquire a new pattern of movement which remains after 2-weeks follow-up period. This outlasting change in motor performance might reflect motor learning (ML) [22]. Indeed, other markers of ML have also been detected. Firstly, movement variability (either for amplitude and cycle duration) did not rise with increasing movement amplitude [23]. Secondly, the increase of movement amplitude was not driven by frequency waning (i.e., amplitudefrequency trade-off). Remarkably, significant improvements in movement amplitude were bilateral in the EG. "Bilateral learning" after "unilateral (right-dominant) practise" has been shown in healthy subjects [24].

The second key finding was the significant increase of the SP-ratio in the EG's LH. In previous studies the lessening of symptoms was associated with SP increasing [25] and [26]. However in our study, the SP-ratio dynamics along the three evaluation-points was different from that of hypometria. LH SP-ratio increased right after training-sessions [5] and [6], but returned to PRE levels at POST2, whereas the effect on hypometria remained after the period without practice. This effect might be following the dynamics of cortical excitability along the different phases of ML [27], [28] and [29].

For the EG and CG the $\mathrm{I} / \mathrm{O}_{\text {ACTIVIATION }}$ slope changed after training. This might be compatible with $\mathrm{ML}$ induced by MP, as they were experienced by the two groups in the LH. However, I/O recruitment became flatter after training, which is different from an increase of the steepness of the recruitment curves as a marker of ML in healthy subjects [30]. We suggest that ML might be differently expressed for PDp because they present reduced inhibition of the cortico-spinal drive during tonic contractile activity.

The differential role of imitation-MP from MP alone was not only observed in SP, but also in MT's. The CG (MP no-imitation) reduced their LH MT's (i.e., increased excitability) after the protocol, in agreement with ML in healthy subjects [30]. This is different in the EG, for whom MT's were unchanged; we suggest this effect might engage physiological mechanisms responsible also for the increased inhibition observed in SP-ratio in this group [31]. It is however also possible that EG and CG were experiencing a similar process of cortical reorganization, but with a faster profile in the case of EG.

After-training changes in excitability of the $\mathrm{H}_{\text {more-affected }}$ and $\mathrm{H}_{\text {less-affected }}$ support the idea that MPimitation produces different adaptation than MP alone. In fact, MT's changed in different directions for both groups, similarly to the LH/RH results; this is perhaps explained by the balanced proportions of $\mathrm{LH} / \mathrm{RH}$ affectation in the two groups of PDp. For the $\mathrm{I} / \mathrm{O}_{\text {ACTIVATION }}$ we only observed changes in the $\mathrm{H}_{\text {less- }}$ affected, again in $\mathrm{CG}$ and $\mathrm{EG}$, suggesting the possibility that $\mathrm{H}_{\text {less-affected }}$ remains more adaptable than $\mathrm{H}_{\text {more- }}$ affected. This reinforces our suggestion that training after-effects on $\mathrm{I} / \mathrm{O}_{\mathrm{ACTIVATION}}$ is due to MP (shared in both groups), and not to imitation. Importantly, training reduced slopes at POST-2 vs. POST, resembling the LH profile in both groups. The SP-ratio was unchanged if considering hemisphere affectation. However the ratio increased significantly in the EG's LH at POST, perhaps driven by right dominant-hand training. These possibilities (relevant for neuro-rehabilitation) must be confirmed in the future.

\subsection{Limitations of the study}

Since this is a pilot-study the small sample size imposes caution in results interpretation. Also, the PDp mostly presented bradykinetic-rigid phenotype in intermediate disease stages. Therefore, our results might be not replicable with some other phenotypes or disease stages.

\section{Conclusion}

The increase in movement amplitude and LH-SP-ratio observed exclusively in PDp trained with MP and imitation reflects that imitation training extended the effects of MP alone. This suggests that motor imitation might be useful as complementary treatment for reducing movement hypometria in PD. These results must be confirmed in future larger trials. 


\section{Authors roles}

1) Research project: A) Conception: VRG, CGS, GS, JF, JC, PA. B) Organization: VRG, PA, JC. C) Execution; VRG, YCB, PA.

2) Statistical Analysis: A) Design: VRG, PA. B) Execution: VRG, PA. C) Review and Critique; VRG, YCB, NE, JC, PA.

3) Manuscript: A) Writing of the first draft: VRG, PA, JC. B) Review and Critique: ALL CONTRIBUTORS.

\section{Financial disclosures of all authors (for the preceding 12 months)}

Grants

VRG and YCB were granted by the FPU-MECD AP2010-2774 \& AP2010-2775 Spain.

\section{Contracts}

NE had a postdoctoral contract in the Department of Medicine at the University of A Coruña.

CGS is neurology specialist at the Movement Disorders Unit, University of A Coruña Hospital.

GS had a research contract in the Department of Electronics and Computer Scienceat the University of Santiago de Compostela.

JF is Professor in the Department of Electronics and Computer Scienceat the University of Santiago de Compostela.

JC is Full Professor of Physiology in the Department of Medicine at the University of A Coruña. Principal Investigator in Projects "Conselleria de Educación 2007/000140-0; Ayudas Grupos Consolidados, Consellería de Educación, 2014; and Dirección Xeral de I + D + i; 2010-2012".

PA is Assistant Professor in the Department of Physical Education and Sport Science at the University of A Coruña.

Financial disclosure/conflict of interest

Nothing to declare.

\section{Fundings sources for study}

This work was supported by Xunta de Galicia (Conselleria de Educación 2007/000140-0; Ayudas Grupos Consolidados, Consellería de Educación, 2014 and Dirección Xeral de I + D + i; 2010-2012), Spain. VRG \& YCB were granted by the FPU-MECD AP2010-2774 \& AP2010-2775 Spain.

\section{Appendix A. Supplementary data}

The following are the supplementary data related to this article: 

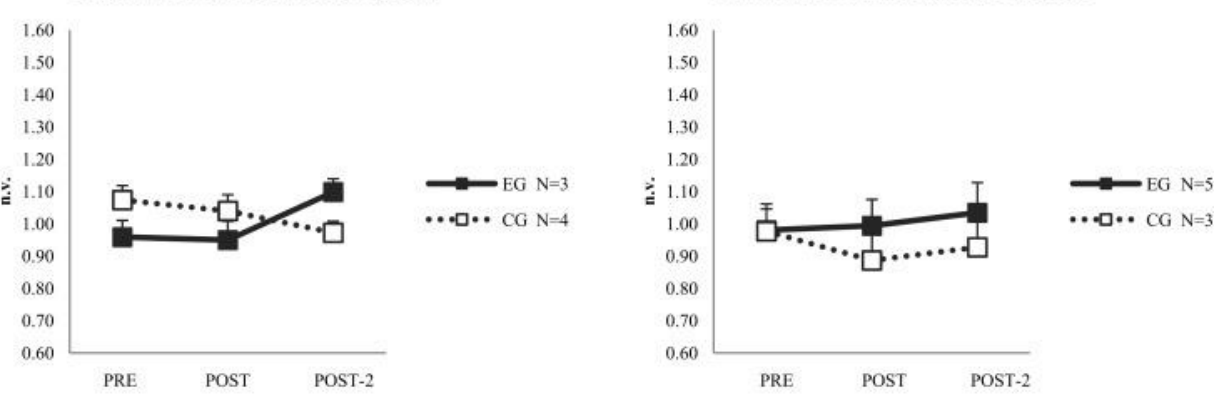

Thresholds in LH in less affected LH patients

Thresholds in RH in less affected RH patients
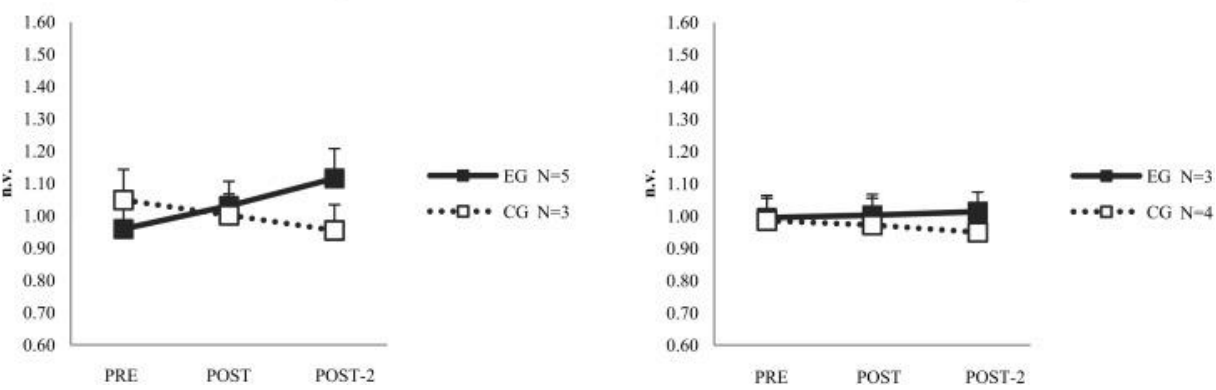

Supplementary Fig 1Training Effects on MT's for Left and Right hemispheres taking into account the degree of affectation (more/less) for LH and RH affected PDp. Only for descriptive information; statistical analysis was not performed due to the fragmentation of the sample size.

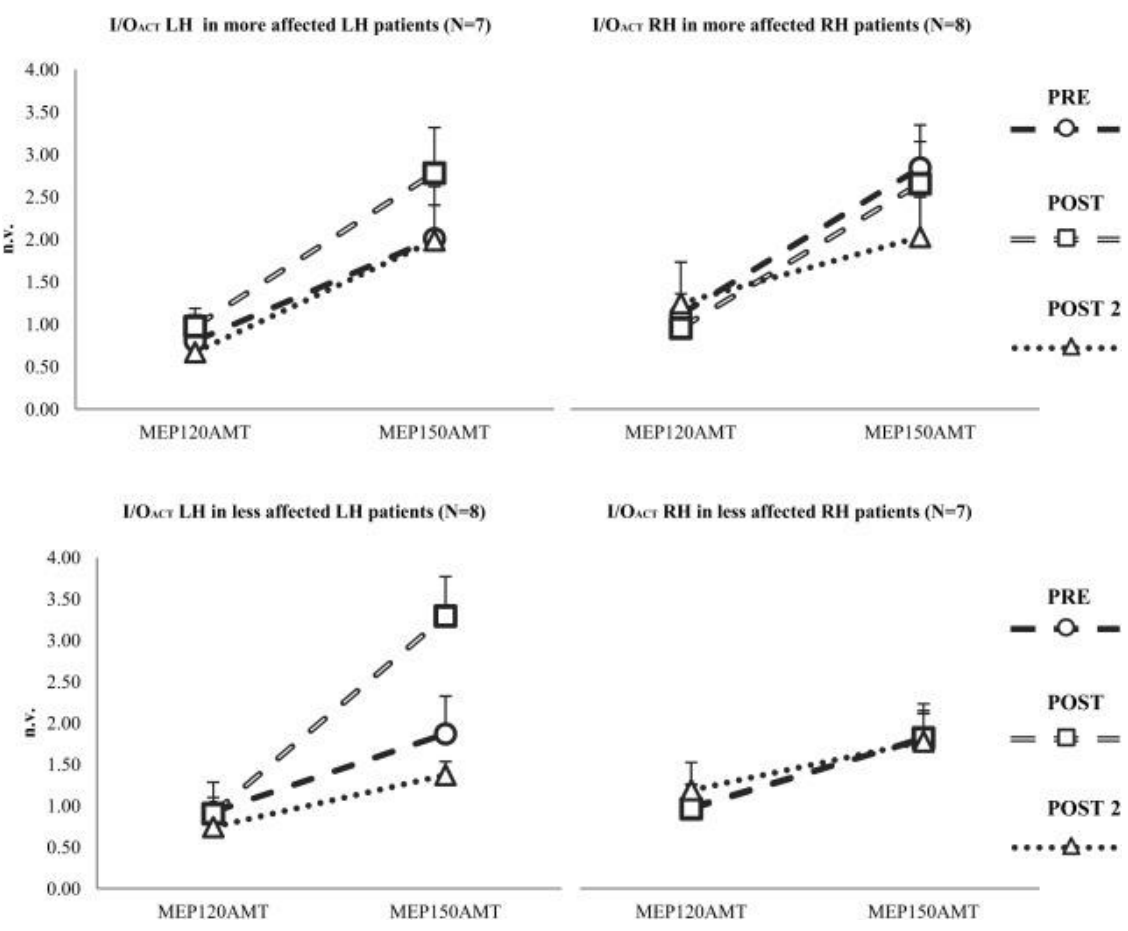

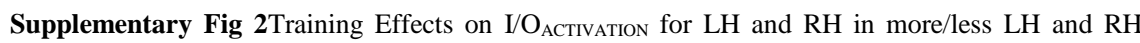
affected PDp. Only for descriptive information; statistical analysis was not performed due to the fragmentation of the sample size. 


\section{References}

[1]. A.J. Espay, J.P. Giuffrida, R. Chen, M. Payne, F. Mazzella, E. Dunn, et al. Differential response of speed, amplitude, and rhythm to dopaminergic medications in Parkinson's disease. Mov. Disord., 26 (2011), pp. 2504 2508 http://dx.doi.org/10.1002/mds.23893.

[2]. R. Cantello, R. Tarletti, C. Civardi. Transcranial magnetic stimulation and Parkinson's disease. Brain Res. Brain Res. Rev., 38 (2002), pp. 309-327.

[3]. U.L.F. Ziemann. Pharmaco-transcranial magnetic stimulation studies of motor excitability. Handb. Clin. Neurol., 116 (2013), pp. 387-397 http://dx.doi.org/10.1016/b978-0-444-53497-2.00032-2.

[4]. S.R. Filipovic, J.C. Rothwell, K. Bhatia. Slow (1 Hz) repetitive transcranial magnetic stimulation (rTMS) induces a sustained change in cortical excitability in patients with Parkinson's disease. Clin. Neurophysiol., 121 (2010), pp. 1129-1137 http://dx.doi.org/10.1016/j.clinph.2010.01.031.

[5]. B.E. Fisher, A.D. Wu, G.J. Salem, J. Song, C.H. Lin, J. Yip, et al. The effect of exercise training in improving motor performance and corticomotor excitability in people with early Parkinson's disease. Arch. Phys. Med. Rehabil., 89 (2008), pp. 1221-1229 http://dx.doi.org/10.1016/j.apmr.2008.01.013.

[6]. M. Mak, M. Hallett. Effect of cued training on motor evoked potential and cortical silent period in people with Parkinson's disease. Clin. Neurophysiol., 124 (2013), pp. 545-550 http://dx.doi.org/10.1016/j.clinph.2012.08.017.

[7]. E. Pelosin, M. Bove, P. Ruggeri, L. Avanzino, G. Abbruzzese. Reduction of bradykinesia of finger movements by a single session of action observation in Parkinson disease. Neurorehabil. Neural Repair, 27 (2013), pp. 552-560 http://dx.doi.org/10.1177/1545968312471905.

[8]. M. Alegre, M.C. Rodríguez-Oroz, M. Valencia, M. Pérez-Alcázar, J. Guridi, J. Iriarte, et al. Changes in subthalamic activity during movement observation in Parkinson's disease: is the mirror system mirrored in the basal ganglia?. Clin. Neurophysiol., 121 (2010), pp. 414-425 http://dx.doi.org/10.1016/j.clinph.2009.11.013.

[9]. V. Robles-García, P. Arias, G. Sanmartín, N. Espinosa, J. Flores, K.L. Grieve, et al. Motor facilitation during real-time movement imitation in Parkinson's disease: a virtual reality study. Parkinsonism Relat. Disord., 19 (2013), pp. 1123-1129 http://dx.doi.org/10.1016/j.parkreldis.2013.08.005.

[10]. S. V Adamovich, G.G. Fluet, A. Mathai, Q. Qiu, J. Lewis, A.S. Merians. Design of a complex virtual reality simulation to train finger motion for persons with hemiparesis: a proof of concept study. J. Neuroeng. Rehabil., 6 (2009), p. 28 http://dx.doi.org/10.1186/1743-0003-6-28

[11]. A.J. Hughes, S.E. Daniel, L. Kilford, A.J. Lees. Accuracy of clinical diagnosis of idiopathic Parkinson's disease: a clinico-pathological study of 100 cases. J. Neurol. Neurosurg. Psychiatry, 55 (1992), pp. 181-184 http://dx.doi.org/10.1136/jnnp.55.3.181.

[12]. R.C. Oldfield. The assessment and analysis of handedness: the Edinburgh inventory. Neuropsycholo., 9 (1971), pp. 97-113 http://www.ncbi.nlm.nih.gov/pubmed/5146491?dopt=Citation.

[13]. C.G. Goetz, S. Fahn, P. Martinez-Martin, W. Poewe, C. Sampaio, G.T. Stebbins, et al. Movement Disorder Society-sponsored revision of the Unified Parkinson's Disease Rating Scale (MDS-UPDRS): Process, format, and clinimetric testing plan. Mov. Disord., 22 (2007), pp. 41-47 http://dx.doi.org/10.1002/mds.21198.

[14]. T. Vanbellingen, B. Kersten, B. Van Hemelrijk, M. Bertschi, R. Mu, W. De Weerdt, et al. Comprehensive assessment of gesture production: a new test of upper limb apraxia (TULIA). Eur. J. Neurol., 17 (2010), pp. 59$66 \mathrm{http} / / / \mathrm{dx}$. doi.org/10.1111/j.1468-1331.2009.02741.x.

[15]. A. Berardelli, J.C. Rothwell, P.D. Thompson, M. Hallett. Pathophysiology of bradykinesia in Parkinson's disease. Brain, 124 (2001), pp. 2131-2146.

[16]. M.P. Lomarev, S. Kanchana, W. Bara-Jimenez, M. Iyer, E.M. Wassermann, M. Hallett. Placebo-controlled study of rTMS for the treatment of Parkinson's disease. Mov. Disord., 21 (2006), pp. 325-331 http://dx.doi.org/10.1002/mds.20713.

[17]. P. Arias, V. Robles-García, G. Sanmartin, J. Flores, J. Cudeiro, G. Sanmartín, et al. Virtual reality as a tool for evaluation of repetitive rhythmic movements in the elderly and Parkinson's disease patients. PLoS One, 7 (2012), p. e30021 http://dx.doi.org/10.1371/journal.pone.0030021.

[18]. J.Y. Li, A.J. Espay, C.A. Gunraj, P.K. Pal, D.I. Cunic, A.E. Lang, et al. Interhemispheric and ipsilateral connections in Parkinson's disease: relation to mirror movements. Mov. Disord., 22 (2007), pp. 813-821 http://dx.doi.org/10.1002/mds.21386.

[19]. P.M. Rossini, A.T. Barker, A. Berardelli, M.D. Caramia, G. Caruso, R.Q. Cracco, et al. Non-invasive electrical and magnetic stimulation of the brain, spinal cord and roots: basic principles and procedures for routine clinical application. Report of an IFCN committee. Electroencephalogr. Clin. Neurophysiol., 91 (1994), pp. 79-92.

[20]. M. Kojovic, P. Kassavetis, M. Bologna, I. Pareés, I. Rubio-Agusti, A. Beraredelli, et al. Transcranial magnetic stimulation follow-up study in early Parkinson's disease: a decline in compensation with disease progression?. Mov. Disord., 30 (2015), pp. 1098-1106 http://dx.doi.org/10.1002/mds.26167.

[21]. C.J. Bohil, B. Alicea, F.A. Biocca. Virtual reality in neuroscience research and therapy. Nat. Rev. Neurosci., 12 (2011), pp. 752-762 http://dx.doi.org/10.1038/nrn3122.

[22]. G.M. Petzinger, B.E. Fisher, S. McEwen, J.A. Beeler, J.P. Walsh, M.W. Jakowec. Exercise-enhanced neuroplasticity targeting motor and cognitive circuitry in Parkinson's disease. Lancet Neurol., 12 (2013), pp. 716-726 http://dx.doi.org/10.1016/s1474-4422(13)70123-6.

[23]. A. Nieuwboer, L. Rochester, L. Muncks, S.P. Swinnen. Motor learning in Parkinson's disease: limitations and potential for rehabilitation. Parkinsonism. Relat. Disord., 15 (Suppl 3) (2009), pp. S53-S58 http://dx.doi.org/10.1016/s1353-8020(09)70781-3. 
[24]. S. Koeneke, C. Battista, L. Jancke, M. Peters. Transfer effects of practice for simple alternating movements. J. Mot. Behav., 41 (2009), pp. 347-355 http://dx.doi.org/10.3200/JMBR.41.4.347-356.

[25]. A.P. Strafella, F. Valzania, S.A. Nassetti, A. Tropeani, A. Bisulli, M. Santangelo, et al. Effects of chronic levodopa and pergolide treatment on cortical excitability in patients with Parkinson's disease: a transcranial magnetic stimulation study., Clin. Neurophysiol.. 111(2000) 1198-1202. http://www.ncbi.nlm.nih.gov/pubmed/10880793 (accessed 21.06.2014).

[26]. J. Däuper, T. Peschel, C. Schrader, C. Kohlmetz, G. Joppich, W. Nager, et al. Effects of subthalamic nucleus (STN) stimulation on motor cortex excitability. Neurolog., 59 (2002), pp. 700-706.

[27]. V. Puttemans, N. Wenderoth, S.P. Swinnen. Changes in brain activation during the acquisition of a multifrequency bimanual coordination task: from the cognitive stage to advanced levels of automaticity. J. Neurosci., 25 (2005), pp. 4270-4278 http://dx.doi.org/10.1523/jneurosci.3866-04.2005.

[28]. N.C. Rogasch, T.J. Dartnall, J. Cirillo, M.A. Nordstrom, J.G. Semmler. Corticomotor plasticity and learning of a ballistic thumb training task are diminished in older adults. J. Appl. Physiol., 107 (2009), pp. 1874-1883 http://dx.doi.org/10.1152/japplphysiol.00443.2009.

[29]. S. Koeneke, K. Lutz, U. Herwig, U. Ziemann, L. Jancke, L. Jäncke. Extensive training of elementary finger tapping movements changes the pattern of motor cortex excitability. Exp. Brain Res., 174 (2006), pp. 199-209 http://dx.doi.org/10.1007/s00221-006-0440-8.

[30]. A. Pascual-Leone, D. Nguyet, L.G. Cohen, J.P. Brasil-Neto, A. Cammarota, M. Hallett. Modulation of muscle responses evoked by transcranial magnetic stimulation during the acquisition of new fine motor skills. J. Neurophysiol., 74 (1995), pp. 1037-1045.

[31]. M. a Perez, L.G. Cohen. Interhemispheric inhibition between primary motor cortices: what have we learned? J. Physiol., 587 (2009), pp. 725-726 http://dx.doi.org/10.1113/jphysiol.2008.166926. 